\title{
EIN MEDAILLENMODELL AUF ADOLPH OCCO II
}

\author{
VON HEINFRIED WISCHERMANN
}

Die Berliner Museen bewahren ein rundes Flachrelief mit dem Brustbild eines lesenden Mannes, das 1904 als Geschenk aus der Sammlung Simon an die Skulpturenabteilung kam (Abb.1). Georg Habich und nach seinem Vorbild E. F. Bange haben das Stück ausführlich beschrieben ${ }^{1}$ :

»Bildnis eines Gelehrten. Halbfigur im Profil nach links. Der Gelehrte, bärtig, mit gelocktem Haar, den Blick sinnend geradeaus gerichtet, hält mit beiden Händen ein aufgeschlagenes Buch. Er trägt einen Mantel mit breitem Pelzkragen. Auf dem Kopfe ein Barett. Auf dem einfach profilierten Rande die Umschrift:

ARTIS QUOD TACEO EST SCULPTORIS CULPA FACESSAT

QUOS EGO PYTHAGORAE TU QUOQUE DISCE MODOS.

Im Bildfelde vertieft eingeschnitten: EIIKYPION THN $\Sigma$ OU MEPIMNAN und auf dem Buche, ebenfalls vertieft: links: OMH/PO rechts: O $\Theta E I / O \Sigma$. Auf der Rückseite eine vertieft geschnittene Signatur: IHA mit Stern $\ll^{2}$ (Abb. 2). Auffallend sind an diesem Medaillenmodell - und um ein solches handelt es sich ohne Zweifel - die ausführlichen Umschriften in lateinischer und griechischer Sprache. Diese enthalten jedoch nicht, wie das bei den meisten Bildnismedaillen der Fall ist ${ }^{3}$, den Namen und die »Aetas«Angabe des Dargestellten.

Im folgenden Beitrag soll - als geringfügiges Beispiel für die Möglichkeiten interdisziplinärer Arbeit - beschrieben werden, wie es möglich war, den Namen, den Beruf und das Alter des unbekannten Homerlesers zu ermitteln.

Erste Hinweise, welche Wege man würde einschlagen können, gaben Habich und Bange. Sie hielten das Stück für ein süddeutsches Modell der ersten Hälfte des 16. Jahrhunderts. Dieser - wie wir sehen werden - zutreffende Datierungs- und Lokalisierungsvorschlag war als Ausgangspunkt vielversprechend. Es war nur ein benanntes

1 G. Habich, Die deutschen Schaumünzen des 16. Jahrhunderis, München 1929, I 1 Nr. 36, Taf. IV 6 ; E. F. Bange, Die Bildwerke in Holz, Stein und Ton. Kleinplastik. (Die Bildwerke des Deutschen Museums IV) Berlin u. Leipzig 1930, S. 63/64, Nr. 5389. Ebda Angaben über Material, Durchmesser, Erhaltung, Herkunft. Ein Lesefehler (Bange las $\Sigma \mathrm{O}$ statt $\Sigma \mathrm{OU}$, da er das schon in mittelalterlichen Handschriften übliche hochgestellte $U$ über $\mathrm{O}$ übersah) ist korrigiert; die lateinische Legende von mir zweizeilig - als Distichon - wiedergegeben. Die Aufnahmen des Modells verdanke ich der Freundlichkeit von Dr. Christian Theuerkauff, Berlin.

2 Es gelang mir nicht, das Monogramm IHA mit Stern aufzulösen; möglicherweise muß man auch unter Drehung des Stücks VHI lesen! Stilistisch steht es, wie schon Habich notierte, dem Kreis des Christoph Weiditz nahe. Vielleicht kommt man über die ausgefallenen Buchstabenformen (Ligaturen) weiter.

3 Zahlreiche Beispiele für Bildnismedaillen mit Angabe von Namen und Alter des Dargestellten in Habichs Standardwerk. 
Porträt desselben Mannes zu finden! Da aber Bildniskataloge deutscher Humanisten des 16. Jahrhunderts nicht in nötiger Vollständigkeit existieren ${ }^{4}$ und da zunächst nicht abzusehen war, wie weit der Kopf Porträtähnlichkeit aufwies, war die Suche erfolglos.

Ein anderer Weg, den Habich wohl schon gesehen und mit Hilfe eines Latinisten verfolgt hatte, führte in die Irre. Thnen war aufgefallen, daß das Wort MEPIMNA bei Homer begegnet - und zwar in dem Homerischen Hymnus auf Merkur 44, 160. Die griechische Wortfolge ist jedoch kein Zitat aus Homer, wie Habich behauptete ${ }^{5}$. Die naheliegende Vermutung, den über den Schriften des »Göttlichen Homer « sinnenden Gelehrten unter den deutschen Homerübersetzern, -kommentatoren und -herausgebern des 16. Jahrhunderts zu suchen, führte zu lehrreicher Lektüre ${ }^{6}$, aber nicht zum gewünschten Ergebnis.

Blieb der Weg über die Legenden. Sie mußten aus so eindeutigen Quellen stammen oder doch so eindeutige Aussagen über den Dargestellten enthalten, daß der Kreis der in Frage kommenden süddeutschen Homerleser einzuengen war. Die klassische Philologie half!

Die griechischen Worte sind Zitat: Bibelzitat! Der Anfang von Psalm 54 (55), 23 lautet in der Septuaginta: EIIPPIYON EMI KYPION THN MEPIMNAN $\Sigma \mathrm{OU}^{7}$; in der Vulgata: IACTA SUPER DOMINUM CURAM TUAM (et ipse te enutriet). »Wirff dein anliegen auff den HErrn/der wird dich versorgen/. . « übersetzte Luther ${ }^{8}$ den Vers, der das Motto, der Wahlspruch des Dargestellten ist.

Die lateinischen Worte, die als elegisches Distichon zu lesen sind, lassen sich folgendermaßen interpungieren und übersetzen: ${ }^{9}$ »Dass ich schweige, gehört zu meinem Beruf (zur Ausübung dessen, was ich gelernt habe); fern sei jede Schuld des Bildhauers! Die Vorschriften des Pythagoras, denen ich folge, lerne auch Du! «

Der Homerleser hatte also einen christlichen Wahlspruch, befolgte aus beruflichen Gründen ein Schweigegebot, d. h. er ließ Namens- und Altersangabe absichtlich fort; er entschuldigte den Bildhauer ${ }^{10}$ und erteilte dem Betrachter einen Rat.

Es ist fast unnötig, darauf hinzuweisen, daß die bekannten Sammlungen von De-

4 In den bekannten Icones-Sammlungen (Th. de Bèze, Les vrais pourtraits, Genf 1581; N. Reusner, Icones, Straßburg 1590; J.-J. Boissard, Icones diversorum hominum, Metz 1591; ders., Icones quinquaginta virorum illustrium, Frankfurt 1597-99) kommt unser Gelehrter nicht vor.

5 G. Habich, a.a.O., S. 10; man vgl. den Homerischen Wortindex von H. Ebeling (Lexicon Homericum, Leipzig 1895).

6 Als Grundlage dienten: G. Finsler, Homer in der Neuzeit von Dante bis Goethe, Leipzig u. Berlin 1912, S. 377 ff.; bes. aber Th. Bleicher, Homer in der deutschen Literatur (1450-1740). Zur Rezeption der Antike und zur Poetologie der Neuzeit. Stuttgart 1972.

7 Den entscheidenden Hinweis auf E. Hatch u. H. A. Redpath (A concordance to the septuagint and the other greek versions of the old testament, Oxford 1897, II S. 911) verdanke ich Dr. F. Preißhofen, Freiburg.

8 Vgl. zu Luthers Psalmübersetzung auch: E. Mülhaupt, D Martin Luthers Psalmen = Auslegung, Göttingen 1962, II S. 236-238.

9 Die brauchbarste, hier wiedergegebene Ubersetzung verdanke ich der Hilfsbereitschaft von Prof. H. Haffter in Zürich, der mir einen Vorschlag von Dr. F. Graf, Rom, mitteilte. Vorschläge erhielt ich auch von Dr. F. Preißhofen und Dr. E. Schäfer, Freiburg.

10 Die Bezeichnung sculptor für einen »Conterfetter auch in einem Brief des Grafen von Nogarolles an Dantiscus. Vgl. J. Kolberg, Archivalisches über Christoph Weiditz, den Meister der Dantiscusmedaillen, in : Archiv für Medaillen- und Plakettenkunde 1 (1913/14) S. 113 ff. 


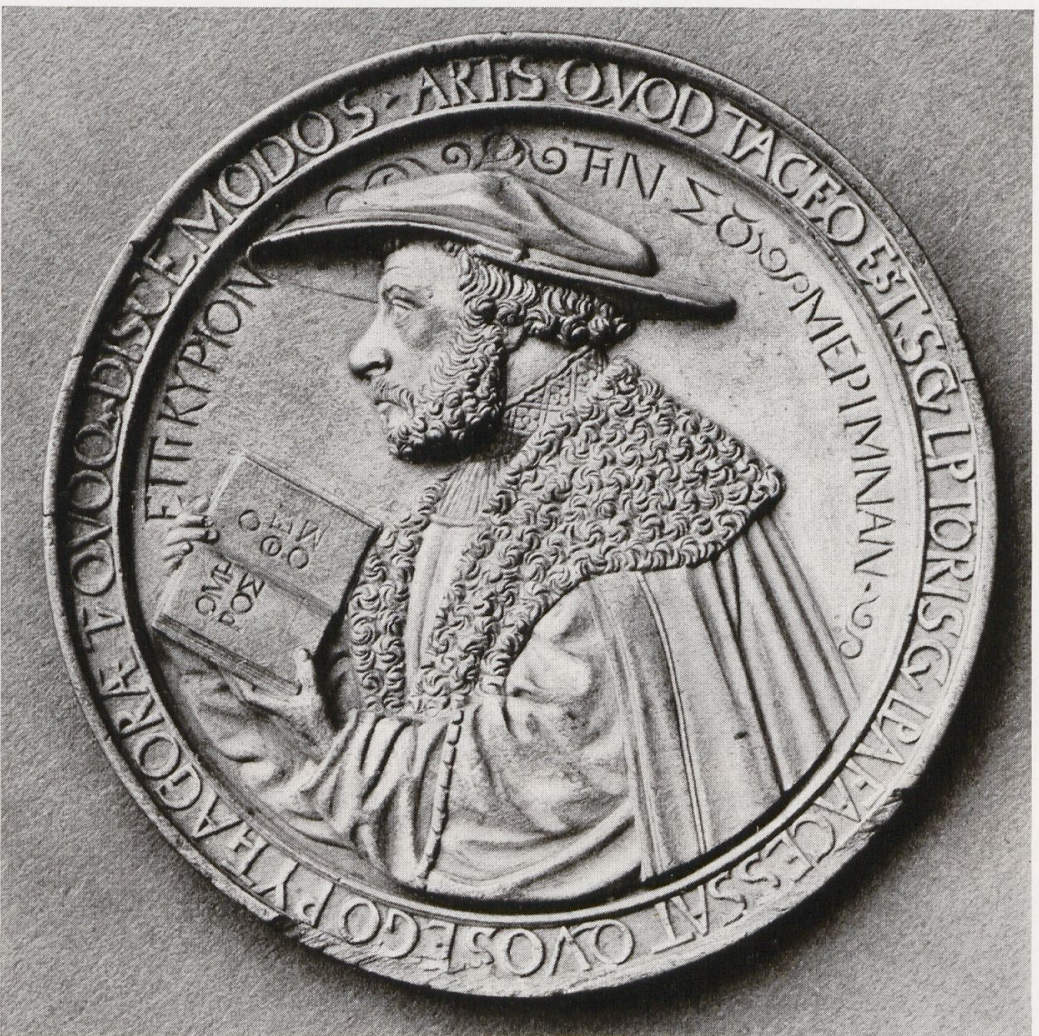

Abb. 1. Medaillenmodell des A. Occo II., Berlin, Staatl. Mus., Skulpturenabteilung. Vorderseite.

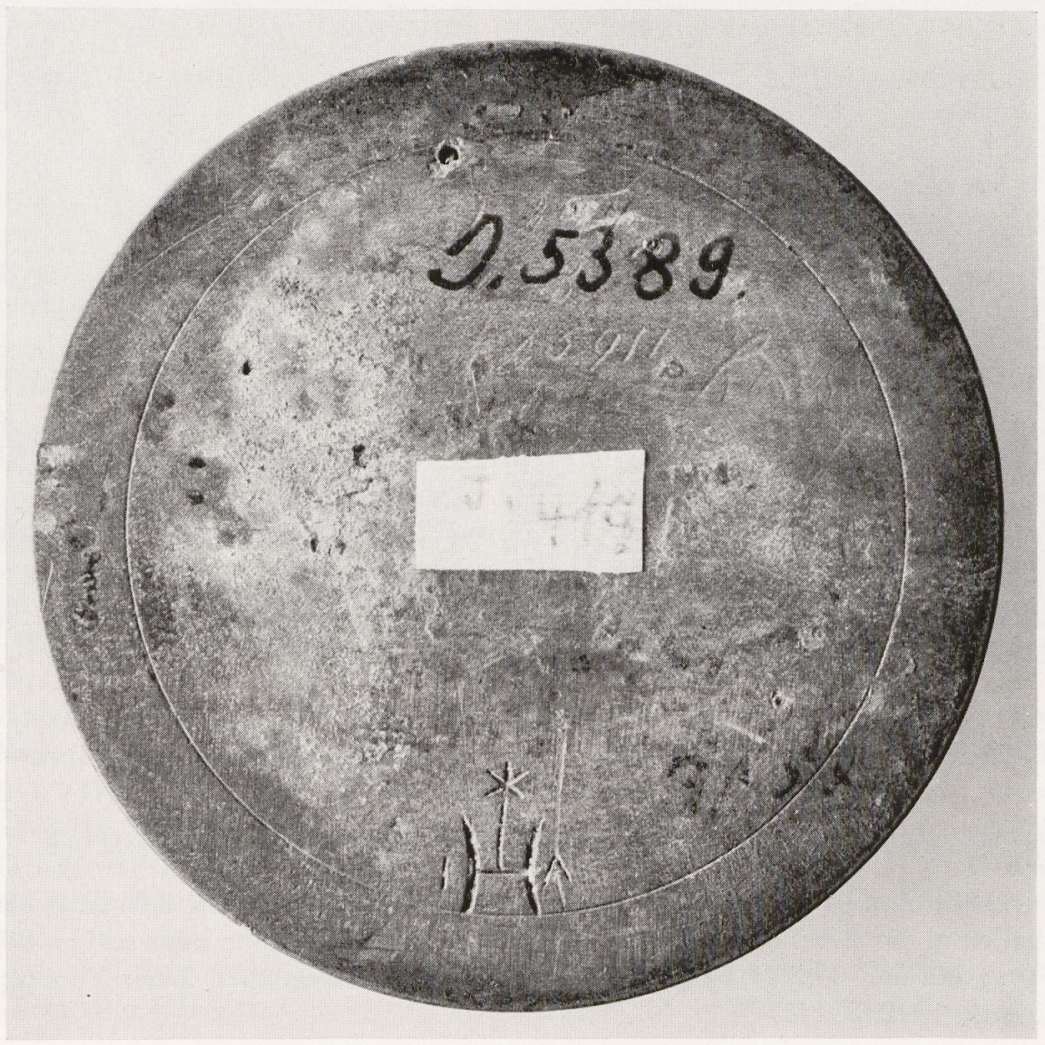

Abb. 2. Medaillenmodell des A. Occo II., Rückseite. 
visen, Motti, Impresen unseren Wahlspruch nicht enthalten ${ }^{11}$. Für die lateinischen Worte war keine Quelle zu ermitteln. Ohnehin war ein Zitat nicht mehr anzunehmen, nachdem die Übersetzung deutlich gemacht hatte, wieviel das Distichon an persönlicher Aussage enthielt. Der Name des Pythagoras-Anhängers fehlte immer noch!

Ein Ausstellungskatalog half weiter und ermöglichte schließlich die endgültige Identifizierung des Gelehrten. Auf der Ausstellung »Augsburger Renaissance《 wurde 1955 eine Medaille gezeigt ${ }^{12}$, deren Rückseite den Psalmvers 54 (55), 23 in der Form IActa CURAM TUAM IN DOMINO NAM ILLI CURA EST DE NOBIS ziert (Abb. 3). Die Vorderseite zeigt ein Bildnis des AdolpHus occo Frisius Medicus ${ }^{13}$, des aus Friesland stammenden Augsburger Stadtarztes Adolph Occo I. (1447-1503). Ein Vergleich der Köpfe macht eine Gleichsetzung der Personen unmöglich: Gesichtsform, Frisur, Bart sind zu verschieden.

Und doch war die Spur richtig. Unser Gelehrter ist ein Occone, ein Mitglied jener »Occonum familia erudita \& clarissima $\ll{ }^{14}$, die mehr als sechs Ärzte mit dem Vornamen Adolphus hervorgebracht hat. Es ist Adolphus Occo II. ${ }^{15}$, der Adoptivsohn des Occo I. Er wurde in Brixen 1494 geboren und im Knabenalter von Occo I., der vielleicht mit ihm verwandt war, an Kindes Statt angenommen. Nach dem Medizinstudium und der Promotion in Bologna ließ er sich in Augsburg nieder, wo er durch seine ärztliche Kunst und seine schriftstellerische Tätigkeit berühmt wurde. 1572 ist er dort gestorben; sein Grabstein ist leider nicht erhalten.

Offensichtlich aus Dankbarkeit und Anhänglichkeit an seinen Adoptivvater, dessen Vor- und Familiennamen er führen durfte, hat er dessen lateinisches Motto in griechischer Version übernommen - ein bislang wohl einmaliger Fall. Schriftquellen, die diese Vermutung stützen, haben sich bisher nicht gefunden. Doch macht ein Porträt von Occo II., das Abraham del Hel 1565 von dem damals einundsiebzigjährigen Arztund Ge-

11 J. v. Radowitz, Die Devisen und Motto des späteren Mittelalters, Stuttgart u. Tübingen 1850; A. Chassant u. H. Tausin, Dictionnaire des devises, Paris 1878; J. Dielietz, Die Wahl- und Denksprüche, Frankfurt 1884; J. Gelli, Divise, motti, imprese ..., Mailand 1916.

12 Katalog »Augsburger Renaissance«, Städt. Kunstsammlungen Augsburg, A. 1955, Nr. 479 mit ält. Lit.

13 Zum Leben der Occonen vgl.: J. Brucker, Historia vitae Adolphorum Occonum, Leipzig 1734; M. Radlkofer, Die humanistischen Bestrebungen der Augsburger Ärzte im 16. Jahrhundert, in: Zeitschrift des Histor. Vereins für Schwaben und Neuburg 20 (1893) S. 25 ff.; G. Gensthaler, Die frühe Medizinalgeschichte der Stadt Augsburg bis zum 16. Jahrhundert. Für Literaturhinweise danke ich Stadtarchivdirektor Dr. F. Blendinger, Augsburg. Zu den Medaillen der Occonen vgl.: G. Habich, Nachtrag zu Christoph Weiditz, in: Archiv für Medaillen- und Plakettenkunde 1 (1913/14) S. 32-34; ders., Die deutschen Schaumünzen, I 1 Nr. 432-436, Taf. LVIII 1-5; H. V. Bühler, Das Ärztegeschlecht der Occo, in: Sudhoffs Archiv für Geschichte der Medizin 28 (1936) S. 14ff.; M. Bernhart, Augsburgs Medailleure und Bildnisse Augsburger Kunsthandwerker auf Schaumünzen des 16. Jahrhunderts, in: Mitteilungen der Bayer. Numismatischen Gesellschaft 55 (1937) S. 70 ff., Taf. XXIII, XXIV 1-2.

14. So nennt sie Jean-Jacques Boissard, Icones quinquaginta . . ., Frankfurt 1597, III S. 13.

15 Zum Leben Occo II. vgl. bes. J. Brucker, a.a.O., S. 34ff. Der Medaillenfreund Goethe gab dem Kanzler v. Müller 1814 eine Empfehlung, die A. Occo über 250 Jahre früher beherzigt hat: »Der Mensch gewöhne sich, täglich in der Bibel oder im Homer zu lesen, oder Medaillen oder schöne Bilder zu schauen, oder gute Musik zu hören«. Vgl. O. Regenbogen, Griechische Gegenwart. Zwei Vorträge über Goethes Griechentum. Leipzig 1942, S. 11. 


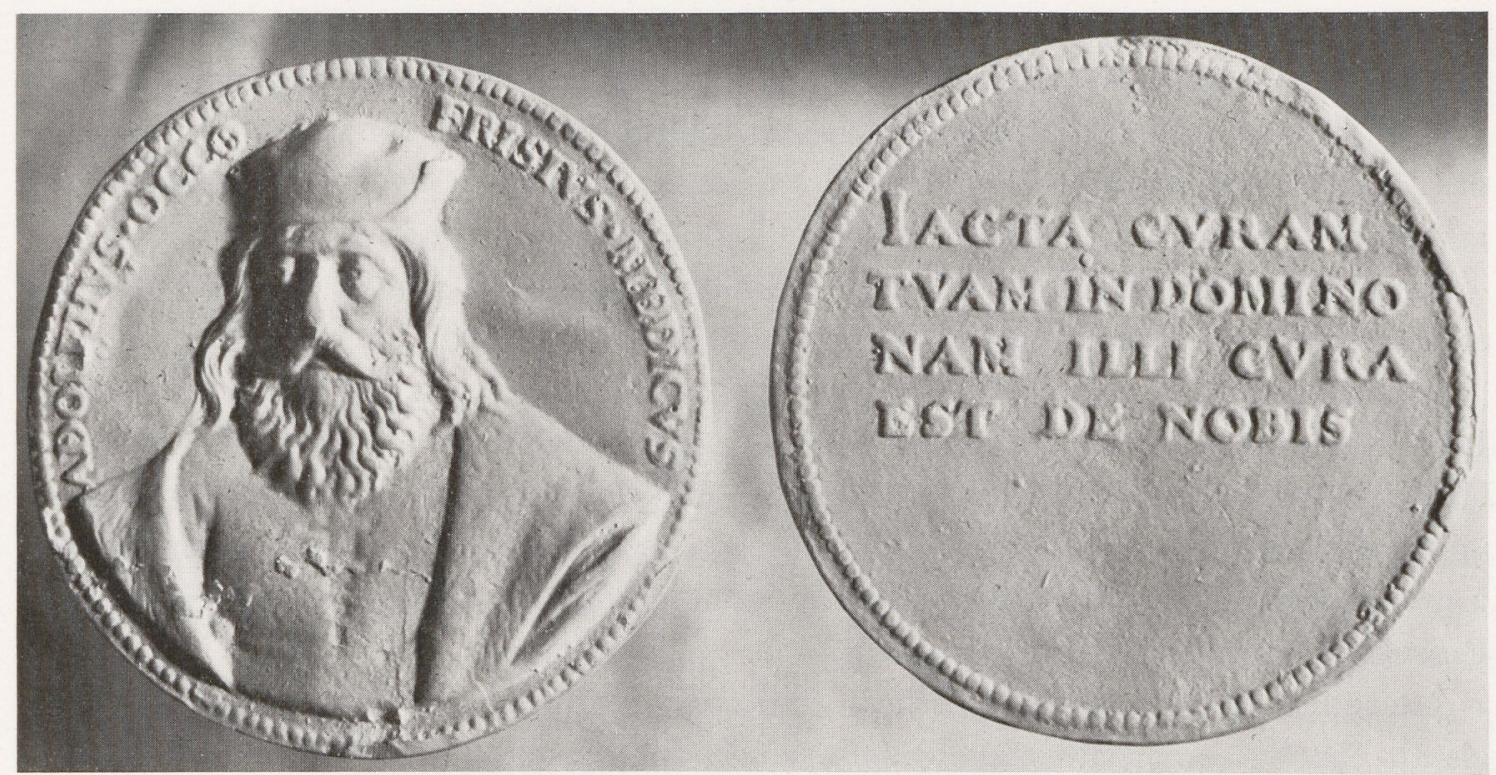

Abb. 3. Medaille des A. Occo I., Augsburg, Städt. Slgn.

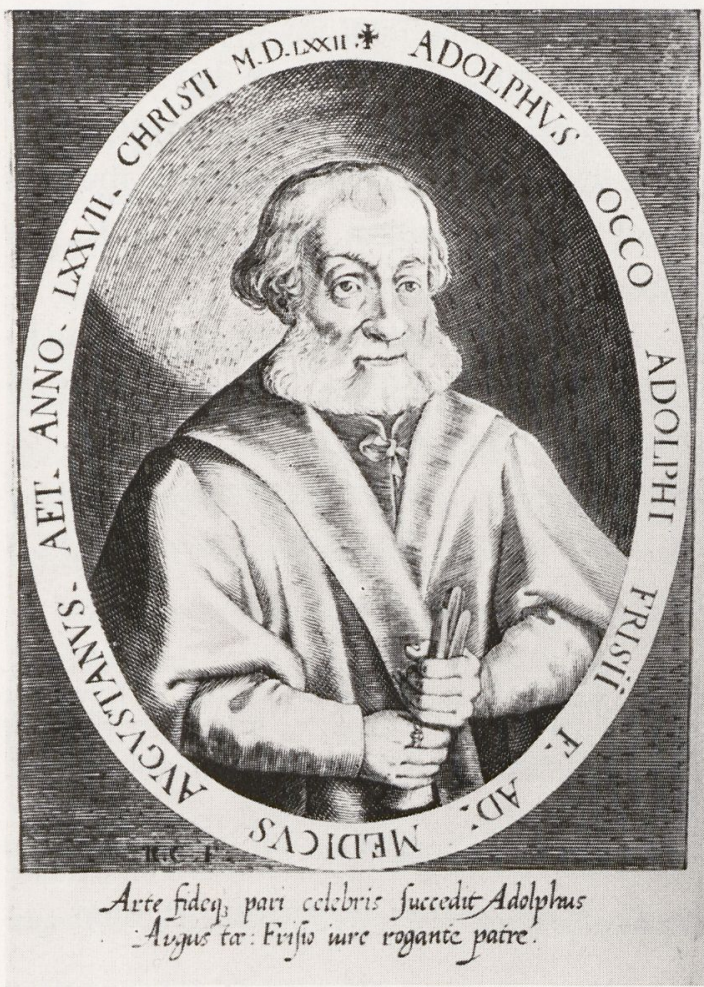

Abb. 4. A. del Hel, Bildnis

des A. Occo II., Augsburg, Städt. Slgn.

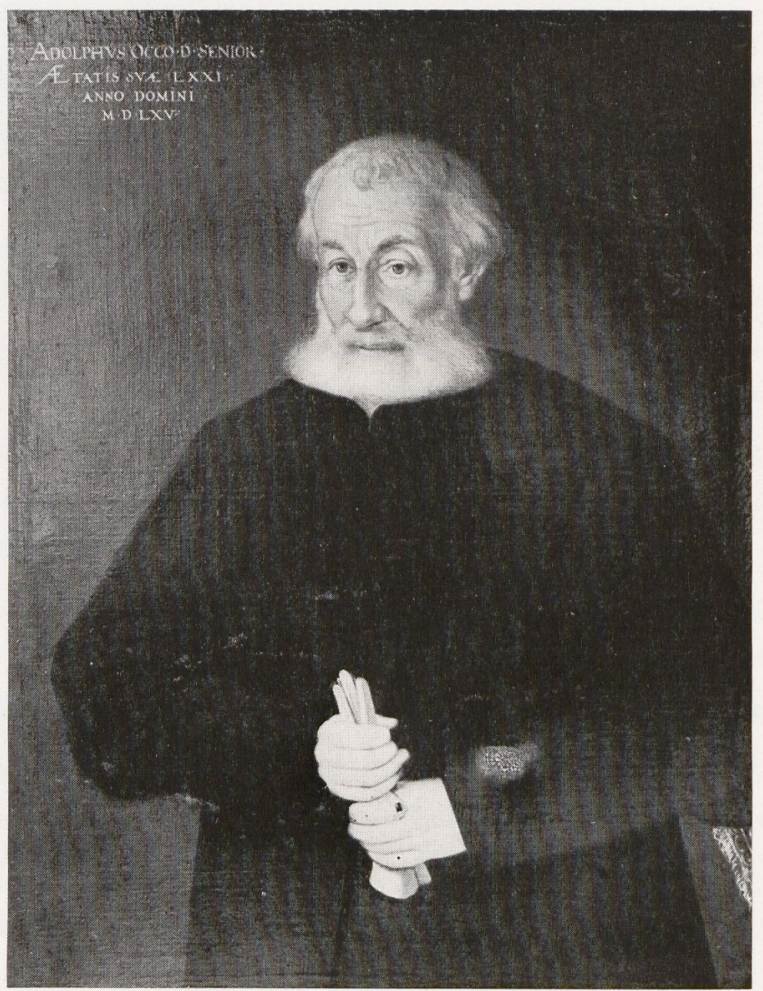

Abb. 5. R. Custos, Bildnis

des A. Occo II., Augsburg, Städt. Slgn.

lehrten malte ${ }^{16}$, die Identifizierung zweifelsfrei (Abb. 4). Die Gesichtsform, vorzüglich die kraftvolle Nase mit den breiten Flügeln und die Schwellung der Wangenknochen, die

16 Ein Stich von R. Custos (Abb. 5), der - wie mir Dr. E. von Knorre, Augsburg, freundlicherweise mitteilte - nach dem Gemälde gemacht wurde, unterstreicht die Identifizierung, da er die Merkmale des 
Frisur, die seitlich die Schläfen freigibt, der Schnitt des Bartes sind unverkennbar gleich. Das Modell zeigt den Humanisten zwanzig, vielleicht fünfundzwanzig Jahre jünger. Es muß gegen 1540 entstanden sein. Letzte Zweifel an der Identifizierung mag das Distichon entkräften, das glänzend auf einen Mediziner paßt (Schweigegebot der ars wegen!).

Unser Medaillenmodell ist demnach in Süddeutschland gearbeitet worden, wohl von einem im zweiten Viertel des 16. Jahrhunderts in Augsburg tätigen Meister.

Eine Bemerkung noch zu Form und Inhalt von Motto und Distichon. Griechische Inschriften auf deutschen Schaumünzen sind nicht selten, man betrachte die Tafeln von Habichs Corpuswerk. Psalmverse treten als Wahlsprüche auf Medaillen keineswegs vereinzelt auf ${ }^{17}$. Über ihre Verwendung - mehrfach auf dem Revers mit einer Darstellung verbunden - ließe sich eine kleine Abhandlung als Beitrag zur Geschichte der Psalmillustration schreiben.

Legenden in Form eines Distichons sind dagegen rar - am Münzrand ist für mehr als die Namens- und Altersangabe kaum Platz. Auf unserem Modell mußte der Psalm-

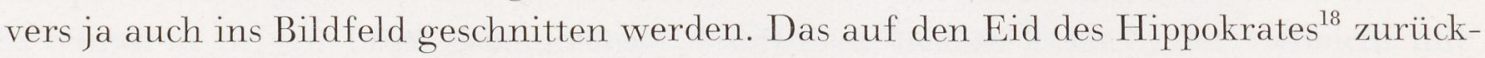
gehende Schweigegebot des Arztes erklärt den Verzicht auf den eigenen Namen; der Name tritt hinter die Tätigkeit zurück. In diesem Zusammenhang ist bemerkenswert, daß Occo II. im Besitz einer Hippokrates-Handschrift war, die er dem befreundeten Humanisten Johann Hagenbut zur Herausgabe seiner lateinischen Hippokrates-Ausgabe lieh $^{19}$. Der Beruf des Arztes und die Neigung zu humanistischen Studien, die sich bei Occo in erstaunlicher Weise verbanden, vererbte er an seinen Sohn Occo III., der ein bedeutender Erforscher der römischen Münzgeschichte werden sollte ${ }^{20}$.

Ungewöhnlich - wenn auch nicht einmalig - ist die Aufforderung an den Betrachter, die pythagoreischen Schweigegebote zu lernen und zu befolgen ${ }^{21}$. Eine undatierte Augsburger Medaille rät zum Bild von zwei Ohren und einem Mund:

》WER OHNE HADER LEBEN WILL / MUSS HÖHREN VIEL / OFFT SCHWEIGEN STILL / / DENN DARUM IST DER MENSCH GEBOHREN/ MIT EINEM MUNDT / UND: ZWEYEN OHREN $\ll{ }^{22}$.

Gesichtes vergröbernd hervorhebt; der Stich schon abgebildet bei H. V. Bühler, a.a.O., Abb. 3. Erwähnt sei, daß die Aufschrift einer gegossenen Medaille auf Occo II. DOMINE VERBUM TUUM FACTUM EST MIHI IN GAUDIUM ebenfalls aus der Bibel stammt: Jer. XV 16; Abb. bei Habich, a.a. O., Taf. LVIII; Bühler, a. a. O., Abb. 7 ; Bernhart, a.a. O., Taf. XXIII 4.

$17 \mathrm{Vgl}$. die von A. Suhle (Die deutsche Renaissance-Medaille. Ein Kulturbild aus der ersten Hälfte des 16. Jahrhunderts, Leipzig 1950) genannten Beispiele.

18 Zum Eid des Hippokrates: F. Büchner, Der Eid des H., Freiburg 1945; W. Capelle, H. Fünf aus erlesene Schriften, Zürich 1955, $209 \mathrm{f}$.

19 Johann Hagenbut (Ianus Comarius) erwähnt dies im Vorwort seiner Hippokrates-Edition, Basel 1546; erwähnt schon bei Brucker, a. a. O., S. 37.

20 Adolphus Occo, Imperatorum Romanorum Numismata A Pompeio Magno Ad Heraclium, Antwerpen 1579.

21 Zum »modus Pythagoricus« vgl.: Aulus Gellius, Noct. Att., lib. I cap. IX; Iamblichus, De vita pythagorica XVI 68, XVII 72, XX 94; Porphyrios, Vita Pythag. 37; Diogenes Laertios, Vita claror. philos. VIII $1 \$ 10$. Allgemein: O. Casel, De veterum philosophorum Graecorum silentio mystico, Giessen 1919, S. 34 .

22 A. von Forster, Die Erzeugnisse der Stempelschneidekunst in Augsburg, Leipzig 1910, Nr. 619: Medaille auf die Verschwiegenheit. 